\title{
The m6A Methyltransferase METTL3 Promotes Cisplatin Resistance and Invasion in Testicular Seminoma via BCL2
}

jingxuan peng ( $\sim$ jingxuan-peng@outlook.com )

Fifth Affiliated Hospital of Sun Yat-sen University https://orcid.org/0000-0002-2772-0564

\section{Yali Xiang}

Fifth Affiliated Hospital of Sun Yat-sen University

Wenfei Lian

Fifth Affiliated Hospital of Sun Yat-sen University

Hanfei Chen

University of Macau

Dongyi Peng

Central South University

Yinghao Yin

Central South University

Qiang Chen

University of Macau

Yuxin Tang

Fifth Affiliated Hospital of Sun Yat-sen University https://orcid.org/0000-0003-4694-558X

\section{Research Article}

Keywords: Seminoma, METTL3, chemosensitivity, m6A, BCL-2

Posted Date: October 18th, 2021

DOI: https://doi.org/10.21203/rs.3.rs-964990/v1

License: (1) This work is licensed under a Creative Commons Attribution 4.0 International License.

Read Full License 


\section{Abstract}

Background: Methyltransferase-like 3 (METTL3) involves in promoting tumor progression through tumorrelated genes N6-methyladenosine (m6A) modification. Our previous study found that METTL3 plays an important role in seminoma chemosensitivity. BCL-2 is a key cause of cisplatin resistance in many tumors. Therefore, we want to explore whether METTL3 affects cisplatin resistance of seminoma by regulating $B C L-2$.

Methods: In this study, we downregulated and overexpressed METTL3 in TCam-2 cisplatin resistant cells (TCam-2/CDDP). Then, m6A RNA methylation quantification of BCL-2 and cell viability assay, cell apoptosis analysis and cell invasion assay were investigated under the condition of with or without cisdichlorodiammine platinum (CDDP) treatment.

Results: Consistent with our previous results, METTL3 significantly affects the chemosensitivity of TCam2/CDDP. After METTL3 downregulated, the proliferation and anti-apoptosis ability of TCam-2/CDDP cells significantly weakened. Correspondingly, overexpression of METTL3 could promote the chemoresistance. However, the phenotype could be partly reversed by decreasing the expression of BCL-2. Moreover, we found that the m6A modification of BCL-2 is more abundant in cisplatin-resistant strains. Knockdown and overexpression of METTL3 significantly affected the m6A modification and the protein level of BCL-2 in TCam-2/CDDP. Finally, we found that METTL3 also promoted the invasion ability of TCam-2/CDDP cells via BCL2.

Conclusion: This study revealed that METTL3 promotes anti-apoptosis and invasion of TCam-2/CDDP through BCL-2. And it indicated that METTL3 and BCL-2 may be an effective treatment target for CDDPresistance seminoma.

\section{Introduction}

Testicular germ cell tumors (TGCTs) are the most commonly diagnosed solid malignancies in young men, and its incidence has been increasing worldwide in recent years [1]. The most common type is the testicular seminoma [2-4]. Furthermore, platinum-based chemotherapy is one of the essential comprehensive adjuvant therapies for TGCTs. However, some patients still cannot achieve remission after receiving platinum-based chemotherapy, which leads to treatment failure and eventually die [5-6]. Therefore, it is very important to explore the mechanism of chemoresistance and find targets for chemoresistant patients.

M6A refers to the methylation of nitrogen at position six of adenine and the most abundant modification in eukaryotic messenger RNAs (mRNAs) [7]. METTL3, an RNA methyltransferase, can promote translation, oncoprotein expression, cell proliferation, survival, and invasion [8]. It has been reported that METTL3 can encourage the translation of target mRNAs in specific cell types and play an essential role in the development of tumors such as bladder cancer, liver cancer, lung cancer, breast cancer and chronic leukemia [7-11]. M6A has a critical regulatory role in tumor stem cells and drug resistance [1],[12]. Our 
previous study found that METTL3 mediates cisplatin resistance through autophagy and modified TFAP2C [13-14] Platinum resistance can also result from defects in initiation of apoptosis,yet the classical anti-apoptosis gene BCL2 have not been proven to be determinants for chemoresistance in germ cell tumors[15-17]. Therefore, this study intends to explore the role of METTL3 in promoting the resistance of TCam-2 cells via classical anti-apoptosis gene BCL2.

\section{Materials And Methods}

\section{Statement}

The Institutional Research Ethics Committee of The Fifth Affiliated Hospital of Sun Yat-sen University approved the study. All experiments adhered to the principles set forth in the Declaration of Helsinki.

\section{Cell culture and Reagents}

The tumor cell lines TCam-2 cells were kindly gifted from Dr. Riko Kitazawa (Department of Diagnostic Pathology, Ehime University Hospital, Matsuyama, Japan). Cells were maintained in complete medium (Roswell Park Memorial Institute Medium-1640 with 10\% fetal calf serum, $100 \mathrm{U} / \mathrm{mL}$ penicillin, and 100 $\mu \mathrm{g} / \mathrm{mL}$ streptomycin) in a humidified $5 \% \mathrm{CO}_{2}, 37^{\circ} \mathrm{C}$ incubator. In our previous study, we established seminoma TCam-2/CDDP cell lines [14]. Cisplatin was purchased from Sigma-Aldrich (St. Louis, MO, USA). Antibodies used in this study were METTL3 (Omnimabs, OM284614), BCL2(Santa Cruz, sc-7382), BCLAF1(Omnimabs, OM272372), MCL1(Omnimabs, OM262629), GAPDH (Abcam, ab125247), Caspase3(Abcam, ab32351), and Cytc(Abcam, ab133504).

\section{M6A RNA Methylation Quantification and MeRIP-qPCR}

M6A RNA Methylation Quantification was performed as previously described [4-5]. Briefly, total RNA is bound to strip wells using an RNA high binding solution. N6-methyladenosine (m6A) is detected using a specific capture N6-methyladenosine antibody and detection antibody. The detected signal is enhanced and then quantified colorimetrically by reading the absorbance in a microplate spectrophotometer at a wavelength of $450 \mathrm{~nm}$. The amount of $\mathrm{m6A}$ is proportional to the OD intensity measured. EZ-Magna RIP kit (Millipore) was used to evaluate the modification level of m6A in the specific gene according to the manufacturer's instructions. The enriched RNA-antibody complex was digested with protease to obtain m6A modified RNA. Then qRT-PCR was used to quantify the enriched RNA. Overall, the RNA containing m6A methylated was coated with m6A antibody and immunoprecipitated on magnetic beads $A / G$.

\section{Western blotting}


Cells were lysed in lysis buffer containing protease inhibitors for 30 minutes. The protein concentration of lysates was determined by the bicinchoninic acid assay (Beyotime Biotechnology). An equivalent amount of protein $(30 \mu \mathrm{g})$ of each sample was separated by $10 \%$ SDS-PAGE and then transferred to PVDF membranes. The membranes were blocked with $5 \%$ non-fat dry milk in $0.2 \%$ Tween- 20 in Tris-buffered saline for one $h$ at room temperature and then probed with primary antibodies. After incubated in the secondary antibody, immunoreactivity was detected by the enhanced chemiluminescence method (Affinity biosciences, Cincinnati, OH, USA ).

\section{Cell viability assay}

The cytotoxic effect of CDDP on TCam-2/CDDP cells was measured by cell count kit 8 (CCK8) assay. TCam-2 cells were seeded into 96-well plates (Corning Incorporated, Corning, NY, USA) at a density of $1{ }^{\star} 10^{\wedge} 4$ cells/well and cultured for 24 hours. Then, cells were cotreated with different concentrations of cisplatin in medium for $0,24,48,72$ hours. Thereafter, $10 \mu \mathrm{L}$ of CCK-8 (Vazyme Biotech, Nanjing, China) solution was added to each well and mixtures were incubated at $37^{\circ} \mathrm{C}$ for additional 1 hour. The optical density (OD) at $450 \mathrm{~nm}$ was read with a microplate reader (Bio-Tek, Winooski, VT, USA).

\section{Cell apoptosis analysis}

Apoptosis was measured by Annexin V-FITC (fluorescein isothiocyanate)/propidium iodide (PI) staining. Briefly, after treatment and incubation for $72 \mathrm{~h}$, cells were collected, washed with PBS, and labeled with Annexin V and propidium iodide in the dark using an Annexin V-FITC apoptosis detection kit (Vazyme Biotech, Nanjing, China). Cell apoptosis was subsequently analyzed by a flow cytometry (Beckman Coulter, Inc. Brea, CA, USA)

\section{Cell invasion assay}

A cell invasion assay was performed using Transwell chambers(Corning, NY, USA) with 8- $\mu$ m pore inserts. Cell suspensions were seeded in the upper chamber, and $500 \mu \mathrm{L}$ of Dulbecco's Modified Eagle's medium containing fetal bovine serum was added to the lower chamber. The noninvading cells were removed with a cotton-tipped swab after 24 hours of incubation, and the invading cells on the bottom surface of the membrane were stained with $0.1 \%$ crystal violet. The invading cells were quantified by counting ten random fields at $\times 200$ magnification.

\section{Quantitative real-time PCR (qPCR)}

Total RNA was extracted from cells using the TRIzol reagent of Vazyme Biotech (Nanjing, People's Republic of China) following the manufacturer's protocol. The PCR primer sequences were as follows: METTL3 forward primer 5'-TTGTCTCCAACCTTCCGTAGT-3', reverse primer 5'- 
CCAGATCAGAGAGGTGGTGTAG-3'『BCL2 forward primer 5'-GGTGGGGTCATGTGTGTGG-3', reverse primer 5'-CGGTTCAGGTACTCAGTCATCC-3'ヌMCL1 forward primer 5'-TGCTTCGGAAACTGGACATCA-3', reverse primer 5'-TAGCCACAAAGGCACCAAAAG-3'; BCLAF1 forward primer 5'- ATGAGACGACCTTATGGGTACA-3', reverse primer 5'- AGAGTGCCTTCTATTCCAGACAG-3'; GAPDH forward primer 5'-

GGAGCGAGATCCCTCCAAAAT-3', reverse primer 5'- GGCTGTTGTCATACTTCTCATGG -3'.

\section{Cell transfection}

SiRNAs for METTL3 and BCL2 were designed. The siRNA sequences were as follows: siRNA-METTL3, sense: 5'-GCAAGUAUGUUCACUAUGATT-3', antisense: 5'-UCAUAGUGAACAUACUUGCAG-3'; siRNA-BCL2, sense: 5'- GCATGCGGCCTCTGTTTGATT-3', antisense: 5'-UCAUAGUGAACAUACUUGCAG-3. Both plasmids and siRNA were transfected with LipofectamineTM 3000 (ThermoFisher, MA, USA) reagent and transfected when the cells met at 70\% confluent. The plasmid overexpressing METTL3 has constructed previously [14].

\section{Data processing}

Data were analyzed with Prism 7 (GraphPad Software) and presented as mean \pm standard deviation (SD). The student's t test was used to analyze the statistical significance of the difference between the two groups. Values are presented as * $p<0.05,{ }^{* *} p<0.01$, and ${ }^{* *} \mathrm{p}<0.001$.

\section{Results}

\section{METTL3 can enhance the anti-apoptosis ability of TCam- 2/CDDP cells}

It has been proved that m6A modification involved in the occurrence and development of tumor drug resistance [17]. We have detected m6A modification of total RNA in TCam-2 cell line and TCam-2/CDDP cell line, and the results showed that the m6A modification in total RNA of TCam-2/CDDP cells was significantly higher than that of TCam-2 cells [13]. Then, siRNA or plasmid was used for knockdown (siMETTL3) or overexpression (OE-METTL3) of METTL3 in TCam-2/CDDP cells, respectively.

The transfection efficiency was validated by qRT-PCR (Figure 1A). Si-METTL3 group and OE-METTL3 group were treated with cisplatin at IC25 concentration [18]. The toxicity of cisplatin to these two groups was detected by CCK8 assay. The results indicated that knockdown of METTL3 significantly inhibited cell viability, while upregulation of METTL3 could accelerate the viability slightly (Figure 1B). As shown in figure 1C and 1D, apoptosis rate was significantly higher in Si-METTL3 group, and lower in OE-METTL3 group compared to NC group. Meanwhile, apoptosis-related proteins Caspase3 and Cytc expression were 
much higher than NC group after METTL3 downregulated (Figure 1E). The aforementioned results reveal that METTL3 increases the chemotherapy resistance of TCam-2 cells through anti-apoptosis.

(A) Relative mRNA of METTL3 in NC, OE-METTL3 and Si-METTL3 groups (B) Cell viability analysis of TCam-2/CDDP treated with CDDP at different time. (C) Cells in different groups were treated with cisplatin for $72 \mathrm{~h}$, followed by staining with apoptosis detection kit and analyzed by flow cytometry. (D) Quantitative summary of the apoptosis rate in the different treatment groups. (E) Western blot analysis of caspase 3 and cytc in the different groups. GAPDH served as the internal control.

\section{METTL3 involves in regulation of BCL2 in TCam-2/CDDP cells}

Researchers have suggested that m6A modification can induce the occurrence of anti-apoptosis in cancers by regulating the expression of classical anti-apoptosis genes [19-20]. In our previous study, we have proved that METTL3 confers resistance to cisplatin in TCam-2 cells [13]. Thus, we speculated that METTL3 could regulate the anti-apoptosis related genes through m6A modification and thus induce chemoresistance. We used m6A-IP-qPCR to evaluate m6A modification of apoptosis-related gene BCL2, BCLAF1 and MCL1. As shown in Figure 2A, the m6A modification of BCL-2 was higher in drug-resistant lines. And the proteins expression of BCL-2 decreased significantly after METTL3 knockdown. The aforementioned results revealed that the higher expression of BCL-2 in TCam-2/CDDP was regulated by METTL3.

\section{METTL3 can promote the anti-apoptosis ability of TCam- 2/CDDP cells via BCL2}

To elucidate the mechanism underlying the anti-apoptosis ability triggered by METTL3, we focused on the BCL2 pathway. As shown in figure $3 \mathrm{~A}$ and $3 \mathrm{~B}$, the BCL2 related m6A modification, and related $\mathrm{mRNA}$ expression significantly decreased after METTL3 knockdown. Furthermore, upregulation of METTL3 strengthened the anti-apoptosis ability of TCam-2/CDDP cells. However, the phenotype could be reversed by downregulation of BCL-2(Figure 3D E). These results illustrate that m6A modification of BCL-2 by METTL3 promotes the chemoresistance of TCam-2/CDDP cells.

\section{METTL3 can also enhance the invasion of TCam-2/CDDP cells via BCL2}

Recent studies have shown that drug-resistant cells have a stronger ability to invade [21-22], and BCL2 has the ability to promote invasion and metastasis [23]. Our results shown that the invasion ability of TCam-2/CDDP cells was much higher after METTL3 overexpressed, and a contrary result was observed when METTL3 knocked down. Meanwhile, the promotion of cell invasion induced by METTL3 upregulation was significantly decreased after BCL2 knockdown (Figure 4). Thus, the result illustrated that METTL3 can enhance the invasion ability of TCam-2 cells via BCL2. 


\section{Discussion}

m6A is the most abundant internal modification in eukaryotic mRNA and plays a vital role in normal biological processes [24-25], includes self-renewal, differentiation of embryonic stem cells, and DNA damage etc [26-39]. Furthermore, emerging evidence has indicated that m6A RNA methylation plays an important role in tumor initiation and progression [40]. Meanwhile, some researchers found mRNA expression levels of VIRMA, YTHDF3, METTL4, ALKBH5 and YTHDC1 were significantly higher in seminoma compared to non- seminoma, which indicated m6A related markers are related to the monitoring and identification of TGCTs (PMID: 30428628). Other researchers' study revealed that the expression of METTL3 increased in TGCTs' cell lines and tissues (PMID: 30903744). METTL3, as m6A writers, involves in many biological processes of tumors as mentioned above. A recent study revealed that ARHG AP5-AS1 stabilized ARHGAP5 mRNA by recruiting METTL3 to stimulate m6A modification of ARHGAP5 mRNA and contribute to drug resistance, indicating that METTL3 was involved in chemotherapy resistance in gastrointestinal cancer [41]. Similarly, mechanistic studies have shown that METTL3 combines with MET and causes the PI3K/AKT signaling pathway to be manipulated, which affects the sensitivity of lung cancer cells to gefitinib [42].

Cisplatin-based chemotherapy plays an important role in the treatment of seminoma. Unfortunately, seminoma is also lethal when gaining resistance to therapy. However, the progress of seminoma and the mechanism of drug resistance are still unclear. More researches on the mechanisms of drug resistance can provide a new direction for clinical treatment. In our previous study, we found that METTL3 was involved in chemoresistance of TCam-2 cells, and promoted CDDP-resistance by m6A modification of ATG5 and TFAP2C. Many researches have revealed that the platinum- resistance often result from defects in initiation of apoptosis, and many studies determine that BCL2, BCLAF1 and MCL1 are important oncogenes involved in cancer by inhibiting apoptosis [15-17]. Therefore, we explored whether METTL3 affected apoptosis to cause drug resistance in seminoma. Likely, we analyzed the apoptosis ratio of TCam-2/CDDP cells cisplatin after CDDP treatment, and found that downregulation of METTL3 obviously alleviated the anti-apoptotic property of cells. These results verify the hypothesis that METTL3 promotes drug resistance of seminoma through decreasing apoptosis.

To further explore the anti-apoptosis mechanism of METTL3, we selected candidate genes via literature retrieval and online database prediction. Through western blot and MeRIP-qPCR, we finally chose BCL-2 as our target. After knockdown of METTL3 in TCam-2/CDDP cells, the mRNA level of BCL-2 was evidently decreased, as well as the m6A abundance. This result revealed that METTL3 could aggrandize the mRNA level of BCL-2 through m6A modification. our results also shown that downregulation of BCL-2 could reverse the effect of METTL3 on anti-apoptosis. The aforementioned results verified METTL3 can promote the anti-apoptosis ability of TCam-2/CDDP cells via m6A modification of BCL2.

However, Metastasis is the worst prognosis predictor in the clinical course of cancer development. Features of metastatic cancer cells include migratory ability, low degree of differentiation, self-renewal and proliferation potentials, as well as resistance to therapies (PMID: 31813120). Although BCL-2 family 
proteins were originally identified as key regulators of apoptosis, an impressive body of evidence has shown that pro-survival members of the BCL-2 family can also promote cell migration, invasion, and cancer metastasis (PMID: 26621844). Our study validated that overexpression of METTL3 enhanced the migration ability of TCam-2/CDDP cells, while siRNA BCL-2 reversed the potential promotion. Therefore, METTL3/BCL-2 participates the migration of TCam-2/CDDP cells.

In summary, we verified the mechanism of METTL3 promoting drug resistance and migration of seminoma through m6A modification of BCL-2 in vitro. However, some limitations should be acknowledged in this study. It remains unclear how METTL3 regulates the mRNA level of BCL-2, and how $\mathrm{BCL}-2$ regulates the apoptosis and migration in seminoma. This study also lacks experiment in vivo to evaluate the conclusions. Furthermore, drug resistance in TGCTs occurs mostly in non-seminoma, the function of METTL3 in non-seminoma needs further study.

\section{Conclusions}

In summary, our study demonstrates the critical role of METTL3 in the process of seminoma resistance, which is characterized by promoting the expression of BCL-2 related proteins or RNA stability to promote the anti-apoptotic ability of TCam-2 cells, and speculated that that METL3 and BCL-2 may be therapeutic targets for drug-resistant seminoma.

\section{Abbreviations}

\section{METTL3}

Methyltransferase-like 3

m6A:N6-methyladenosine

TCam-2/CDDP

TCam-2 cisplatin resistant cells

CDDP

cis-dichlorodiammine platinum

TGCTs

Testicular germ cell tumors

mRNAs

messenger RNAs.

\section{Declarations}

\section{Acknowledgements}

The tumor cell lines TCam-2 cells were kindly gifted from Dr. Riko Kitazawa (Department of Diagnostic Pathology, Ehime University Hospital, Matsuyama, Japan)

\section{Author contributions}


YXT and YLX designed the study; JXP, WFL, YHY and HFC performed the experiments, analyzed data and prepared the figures; DYP, YHY and QC contributed to drafting the manuscript. All authors read and approved the fnal manuscript.

\section{Funding}

This study was supported by the Science and Technology Innovation Bureau of Zhuhai grants to WL (\#20191210E030071) and the Natural Science Foundation of Hunan Province grants to YT (\#2019JJ80097).

\section{Data availability statement}

The data used in the current study are available from the corresponding author on reasonable request.

\section{Availability of data and materials}

The data supporting the conclusions of this study are included within the article.

\section{Ethics approval and consent to participate}

The present study was approved by the Institutional Research Ethics Committee of The Fifth Affiliated Hospital of Sun Yat-sen University (Approval No. L020-1). Consent for publication Not applicable.

\section{Competing interests}

The authors declare that there were no conficts of interest.

\section{References}

1. Einhorn LH. Curing metastatic testicular cancer. Proc Natl Acad Sci U S A. 2002;2(7):4592-5. 99(.

2. Miller KD, Siegel RL, Lin CC, et al. Cancer treatment and survivorship statistics, CA: a cancer journal for clinicians, 2016. CA Cancer J Clin. 2016;66(4):271-89.

3. Trabert B, Chen J, Devesa SS, et al. International patterns and trends in testicular cancer incidence, overall and by histologic subtype, 1973-2007. Andrology. 2015;3(1):4-12.

4. Huyghe E, Matsuda T, Thonneau P. Increasing incidence of testicular cancer worldwide: a review. The Journal of urology. 2003;170(1):5-11. https://doi.org/10.1097/01.ju.0000053866.68623.da.

5. Squillante CM, Vaughn DJ. Targeted therapies in germ cell tumors. Urol Oncol. 2015;33(8):363-9.

6. Horwich A, Shipley J, Huddart R. Testicular germ-cell cancer. Lancet. 2006;4(9512):754-65. 367(.

7. Cheng M, Sheng L, Gao Q, et al. The m 6 A methyltransferase METTL3 promotes bladder cancer progression via AFF4/NF-kB/MYC signaling network. Oncogene. 2019;38(19):3667-80.

8. Lin S, Choe J, Du P, et al. The m(6)A methyltransferase METTL3 promotes translation in human cancer cells. Mol Cell. 2016;5(3):335-45. 62(. 
9. Ma JZ, Yang F, Zhou CC, et al. METTL14 suppresses the metastatic potential of hepatocellular carcinoma by modulating $\mathrm{N}(6)$-methyladenosine-dependent primary microRNA processing. Hepatology. 2017;65:529-43.

10. Cai X, Wang X, Cao C, et al. HBXIP-elevated methyltransferase METTL3 promotes the progression of breast cancer via inhibiting tumor suppressor let-7g. Cancer Lett. 2018;28:415:11-9.

11. Barbieri I, Tzelepis K, Pandolfini L, et al. Promoter-bound METTL3 maintains myeloid leukaemia by m(6)A-dependent translation control. Nature. 2017;552:126-31.

12. Liu A, Cheng L, Du J, et al. Diagnostic utility of novel stem cell markers SALL4, OCT4, NANOG, SOX2, UTF1, and TCL1 in primary mediastinal germ cell tumors. Am J Surg Pathol. 2010;34(5):697-706.

13. Chen $H$, Xiang $Y$, Yin Y, Peng J, Peng D, Li D, Kitazawa R, Tang Y, Yang J. The m6A methyltransferase METTL3 regulates autophagy and sensitivity to cisplatin by targeting ATG5 in seminoma. Transl Androl Urol. 2021 Apr;10(4):1711-1722. doi: 10.21037/tau-20-1411. PMID: 33968659; PMCID: PMC8100844.

14. Wei J, Yin Y, Zhou J, Chen H, Peng J, Yang J, Tang Y. METTL3 potentiates resistance to cisplatin through $\mathrm{m}^{6}$ A modification of TFAP2C in seminoma. J Cell Mol Med. 2020 Oct;24(19):11366-80. doi:10.1111/jcmm.15738. Epub 2020 Aug 28. PMID: 32857912; PMCID: PMC7576266.

15. Squillante CM, Vaughn DJ. Targeted therapies in germ cell tumors. Urol Oncol. 2015;33(8):363-9.

16. Mayer F, Stoop H, Scheffer GL, et al. Molecular determinants of treatment response in human germ cell tumors. Clin Cancer. 2003;9:767-73.

17. Pekarsky Y, Balatti V, Croce CM, et al. BCL2 and miR-15/16: from gene discovery to treatment. Cell Death Differ. 2018;25(1):21-6.

18. Zhou L, Wang HF, Ren HG, Chen D, Gao F, Hu QS, Fu C, Xu RJ, Ying Z, Wang GH. Bcl-2-dependent upregulation of autophagy by sequestosome 1/p62 in vitro. Acta Pharmacol Sin. 2013 May;34(5):651-6. doi:10.1038/aps.2013.12. Epub 2013 Apr 8. PMID: 23564079; PMCID: PMC3647217.

19. Cidado J, Boiko S, Proia T, Ferguson D, Criscione SW, San Martin M, Pop-Damkov P, Su N, Roamio Franklin VN, Sekhar Reddy Chilamakuri C, D'Santos CS, Shao W, Saeh JC, Koch R, Weinstock DM, Zinda M, Fawell SE, Drew L. AZD4573 Is a Highly Selective CDK9 Inhibitor That Suppresses MCL-1 and Induces Apoptosis in Hematologic Cancer Cells. Clin Cancer Res. 2020 Feb 15;26(4):922-934. doi: 10.1158/1078-0432.CCR-19-1853. Epub 2019 Nov 7. PMID: 31699827.

20. Ashkenazi A, Fairbrother WJ, Leverson JD, Souers AJ. From basic apoptosis discoveries to advanced selective BCL-2 family inhibitors. Nat Rev Drug Discov. 2017 Apr;16(4):273-84. doi:10.1038/nrd.2016.253. Epub 2017 Feb 17. PMID: 28209992.

21. Alexander S, Friedl P. Cancer invasion and resistance: interconnected processes of disease progression and therapy failure. Trends Mol Med. 2012 Jan;18(1):13-26. doi:10.1016/j.molmed.2011.11.003. Epub 2011 Dec 15. PMID: 22177734.

22. Jin L, Chun J, Pan C, Alesi GN, Li D, Magliocca KR, Kang Y, Chen ZG, Shin DM, Khuri FR, Fan J, Kang S. Phosphorylation-mediated activation of LDHA promotes cancer cell invasion and tumour 
metastasis. Oncogene. 2017 Jul 6;36(27):3797-3806. doi: 10.1038/onc.2017.6. Epub 2017 Feb 20. PMID: 28218905; PMCID: PMC5501759.

23. Kim EM, Jung CH, Kim J, Hwang SG, Park JK, Um HD. The p53/p21 Complex Regulates Cancer Cell Invasion and Apoptosis by Targeting Bcl-2 Family Proteins. Cancer Res. 2017 Jun 1;77(11):30923100.

24. Roundtree IA, Evans ME, Pan T, et al. Dynamic RNA modifications in gene expression regulation. Cell. 2017;15(7):1187-200. 169).

25. Zhao BS, Roundtree IA, He C. Post-transcriptional gene regulation by mRNA modifications. Nat Rev Mol Cell Biol. 2017;18(1):31-42.

26. Zheng G, Dahl JA, Niu Y, et al. ALKBH5 is a mammalian RNA demethylase that impacts RNA metabolism and mouse fertility. Mol Cell. 2013;10(1):18-29. 49 ).

27. Wang Y, Li Y, Toth Jl, et al. N6-methyladenosine modification destabilizes developmental regulators in embryonic stem cells. Nat Cell Biol. 2014;16(2):191-8.

28. Dominissini D, Moshitch-Moshkovitz S, Schwartz S, et al. Topology of the human and mouse m6A RNA methylomes revealed by m6A-seq. Nature. 2012;29(7397):201-6. 485 ).

29. Meyer KD, Patil DP, Zhou J, et al. 5' UTR m(6)A promotes Cap-independent translation. Cell. 2015;5(4):999-1010. 163 ).

30. Zhao X, Yang Y, Sun BF, et al. FTO-dependent demethylation of N6-methyladenosine regulates mRNA splicing and is required for adipogenesis. Cell Re. 2014;24(12):1403-19.

31. Hsu PJ, Zhu Y, Ma H, et al. Ythdc2 is an N6-methyladenosine binding protein that regulates mammalian spermatogenesis. Cell Res. 2017;27(9):1115-27.

32. Liu N, Dai Q, Zheng G, et al. N(6)-methyladenosine-dependent RNA structural switches regulate RNAprotein interactions. Nature. 2015;26(7540):560-4. 518 ).

33. Alarcon CR, Lee H, Goodarzi $\mathrm{H}$, et al. N6-methyladenosine marks primary microRNAs for processing. Nature. 2015;26(7544):482-5. 519).

34. Geula S, Moshitch-Moshkovitz S, Dominissini D, et al. Stem cells. m6A mRNA methylation facilitates resolution of naïve pluripotency toward differentiation. Science. 2015;27(6225):1002-6. 347 ).

35. Chen T, Hao YJ, Zhang Y, et al. m(6)A RNA methylation is regulated by microRNAs and promotes reprogramming to pluripotency. Cell Stem Cell. 2015;5(3):289-301. 16 ).

36. Xiang $\mathrm{Y}$, Laurent $\mathrm{B}, \mathrm{Hsu} \mathrm{CH}$, et al. RNA m6A methylation regulates the ultraviolet-induced DNA damage response. Nature. 2017;23(7646):573-6. 543 ).

37. Zhao BS, Wang X, Beadell AV, et al. m6A-dependent maternal mRNA clearance facilitates zebrafish maternal-to-zygotic transition. Nature. 2017;23(7642):475-8. 542 ).

38. Wang X, Zhao BS, Roundtree IA, et al. N(6)-methyladenosine modulates messenger RNA Translation Efficiency. Cell. 2015;4(6):1388-99. 161 ).

39. Niu Y, Zhao X, Wu YS, et al. N6-methyl-adenosine (m6A) in RNA: an old modification with a novel epigenetic function. Genom Proteom Bioinform. 2013;11(1):8-17. 
40. Pan Y, Ma P, Liu Y, Li W, Shu Y. Multiple functions of $m^{6} A$ RNA methylation in cancer. J Hematol Oncol. 2018 Mar 27;11(1):48. doi: 10.1186/s13045-018-0590-8. PMID: 29587823; PMCID:

PMC5870302.

41. Zhu L, Zhu Y, Han S, Chen M, Song P, Dai D, Xu W, Jiang T, Feng L, Shin VY, Wang X, Jin H. Impaired autophagic degradation of IncRNA ARHGAP5-AS1 promotes chemoresistance in gastric cancer. Cell Death Dis. 2019 May 16;10(6):383. doi: 10.1038/s41419-019-1585-2. PMID: 31097692; PMCID: PMC6522595.

42. Gao F, Wang Q, Zhang C, Zhang C, Qu T, Zhang J, Wei J, Guo R. RNA methyltransferase METTL3 induces intrinsic resistance to gefitinib by combining with MET to regulate PI3K/AKT pathway in lung adenocarcinoma. J Cell Mol Med. 2021 Mar;25(5):2418-25. doi:10.1111/jcmm.16114. Epub 2021 Jan 24. PMID: 33491264; PMCID: PMC7933928.

\section{Figures}


A
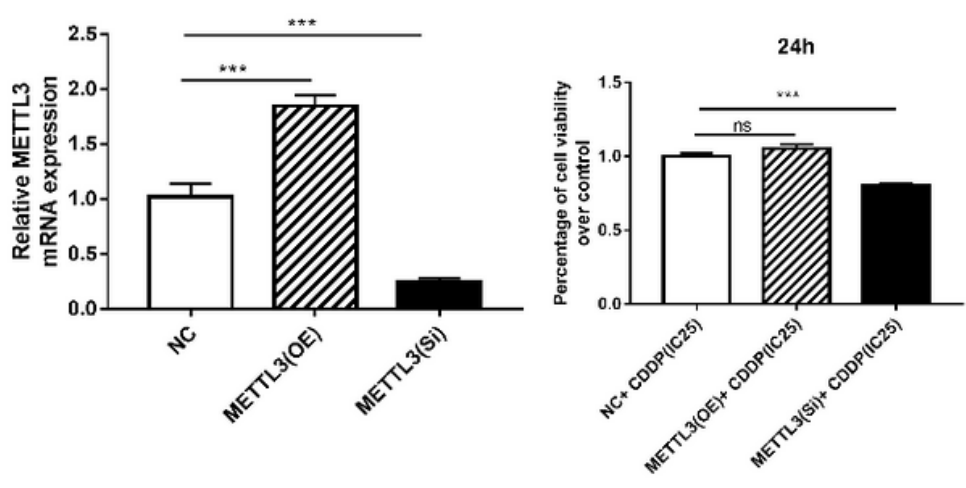

C

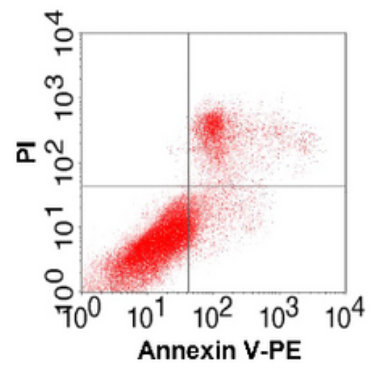

NC+CDDP(IC25)

E

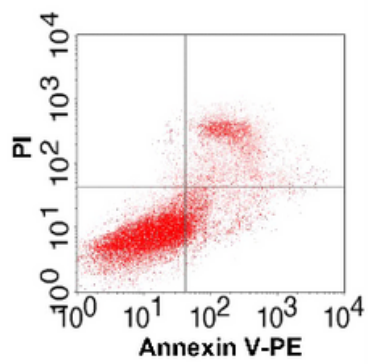

METTL3(OE)+CDDP(IC25)
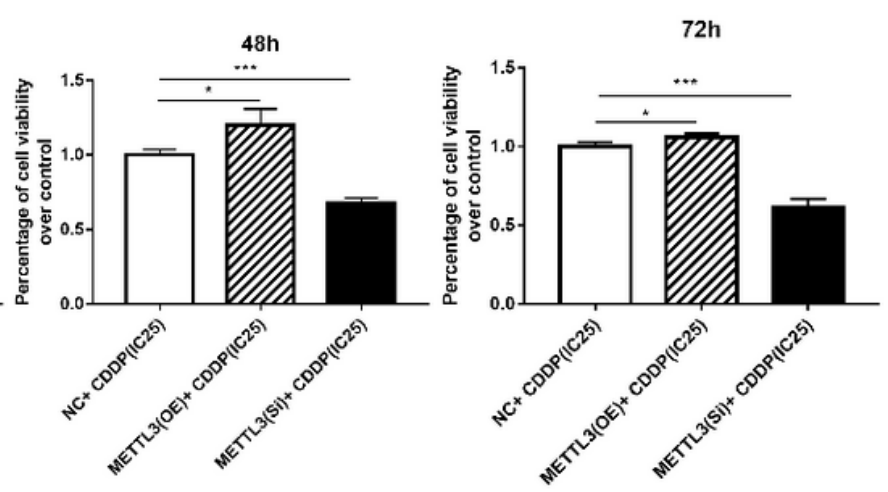

D

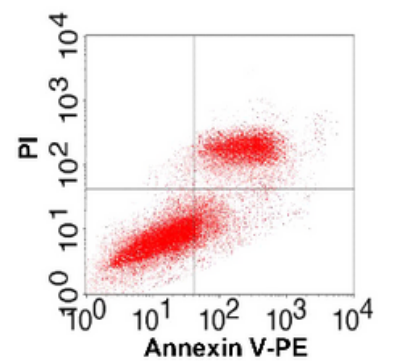

METTL3(Si)+CDDP(IC25)

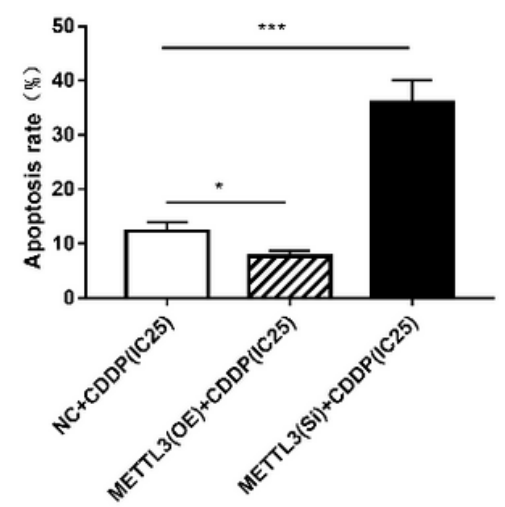

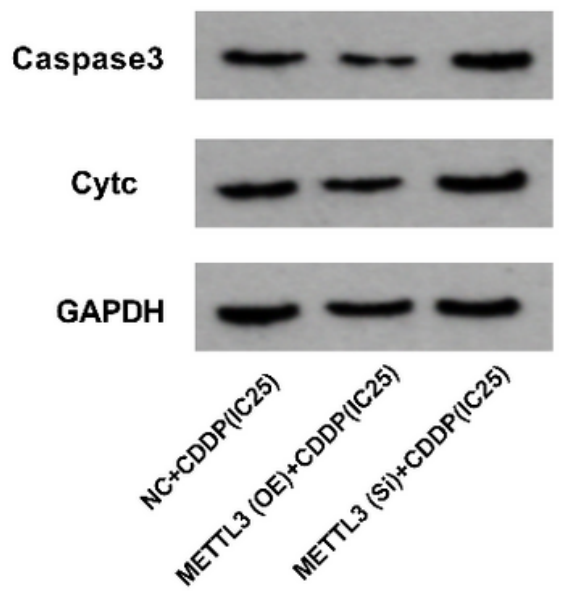
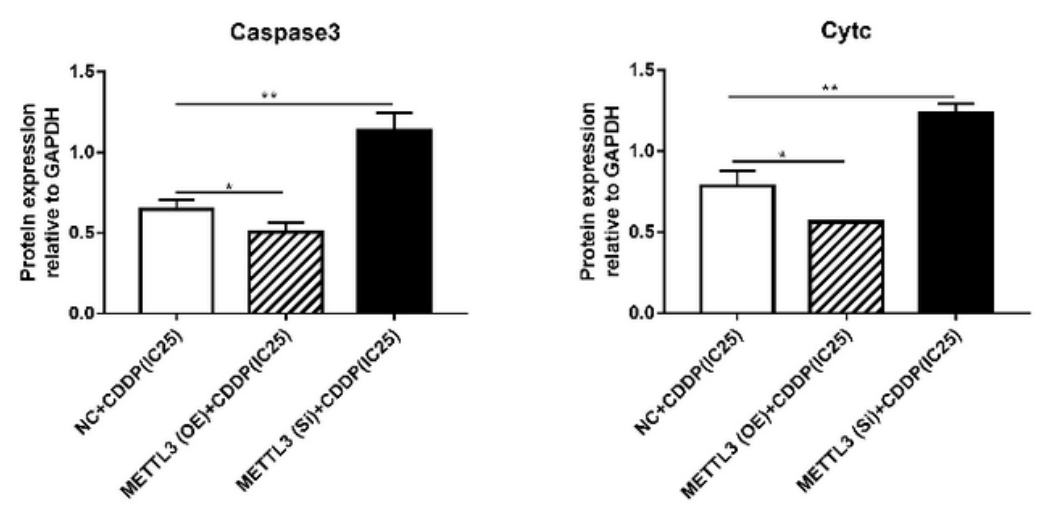

Figure 1

METTL3 enhances the anti-apoptosis ability of TCam-2/CDDP cells (A) Relative mRNA of METTL3 in NC, OE-METTL3 and Si-METTL3 groups (B) Cell viability analysis of TCam-2/CDDP treated with CDDP at different time. (C) Cells in different groups were treated with cisplatin for $72 \mathrm{~h}$, followed by staining with apoptosis detection kit and analyzed by flow cytometry. (D) Quantitative summary of the apoptosis rate in the different treatment groups. (E) Western blot analysis of caspase 3 and cytc in the different groups. GAPDH served as the internal control. 
A

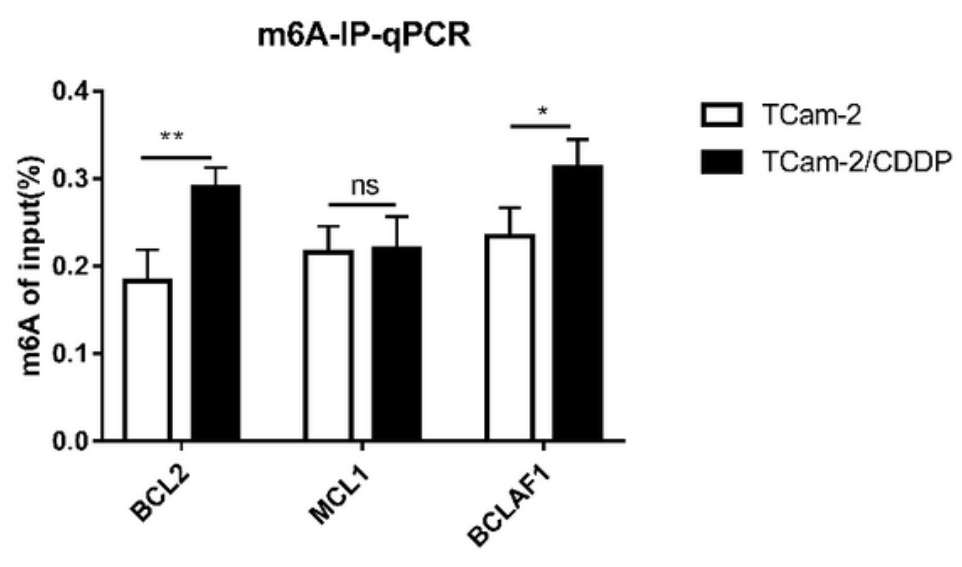

B

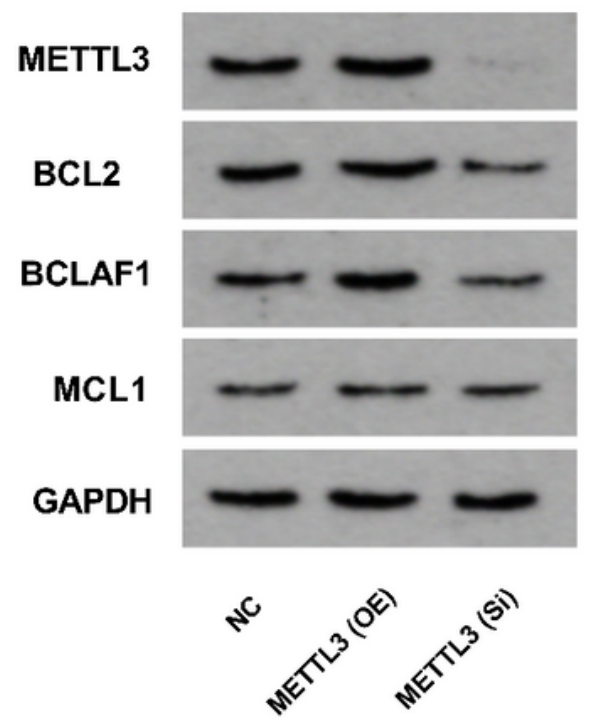

C
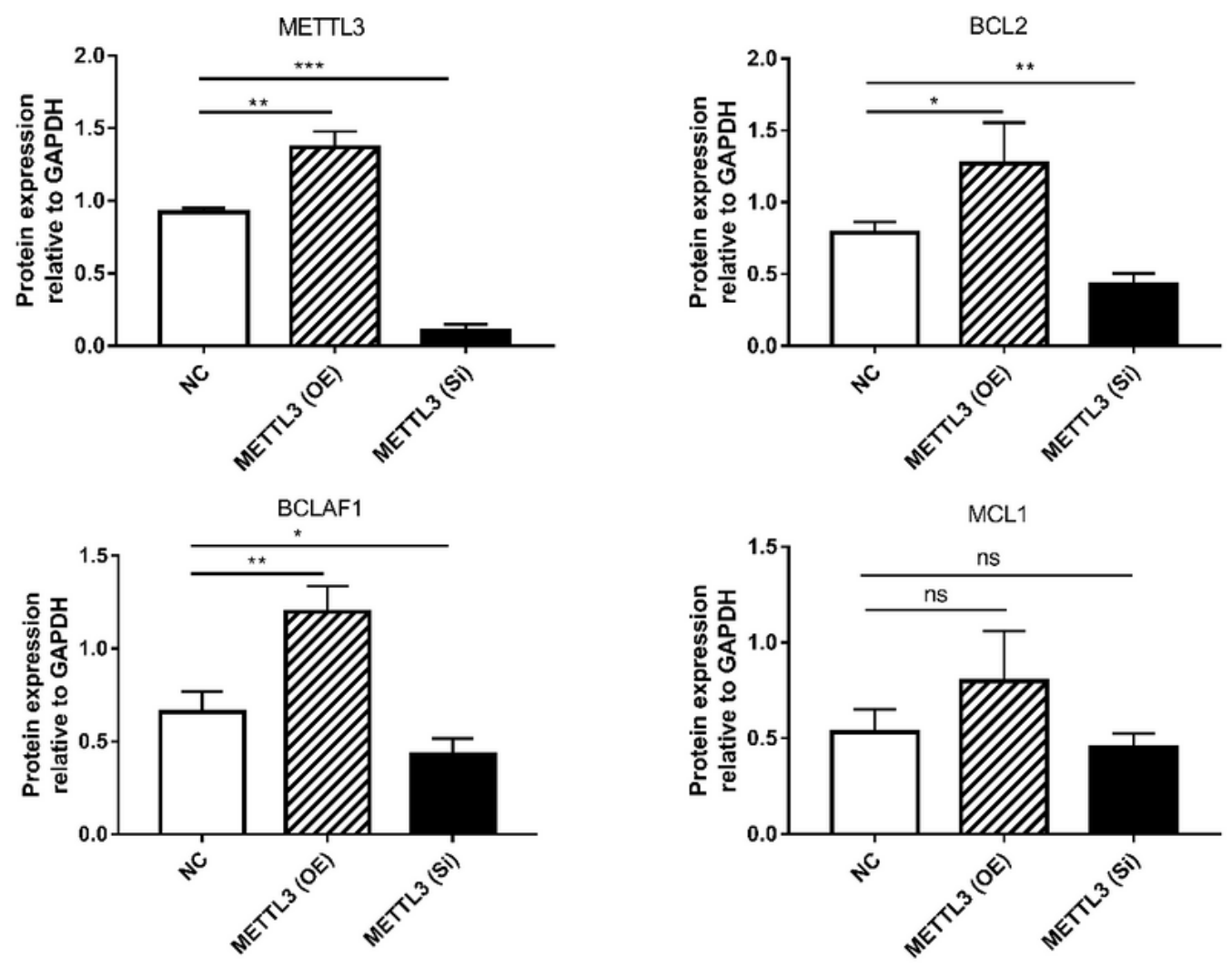

Figure 2

METTL3 involves in the m6A modification of BCL2 in TCam-2/CDDP cells. (A)M6A-IP-qPCR tested the m6A modification of BCL2, MCL1 and BCLAF1. (B, C) Western blot analysis of METTL3, BCL2, BCLAF1 and MCL1 in the different treatment groups. GAPDH served as the internal control. 
A
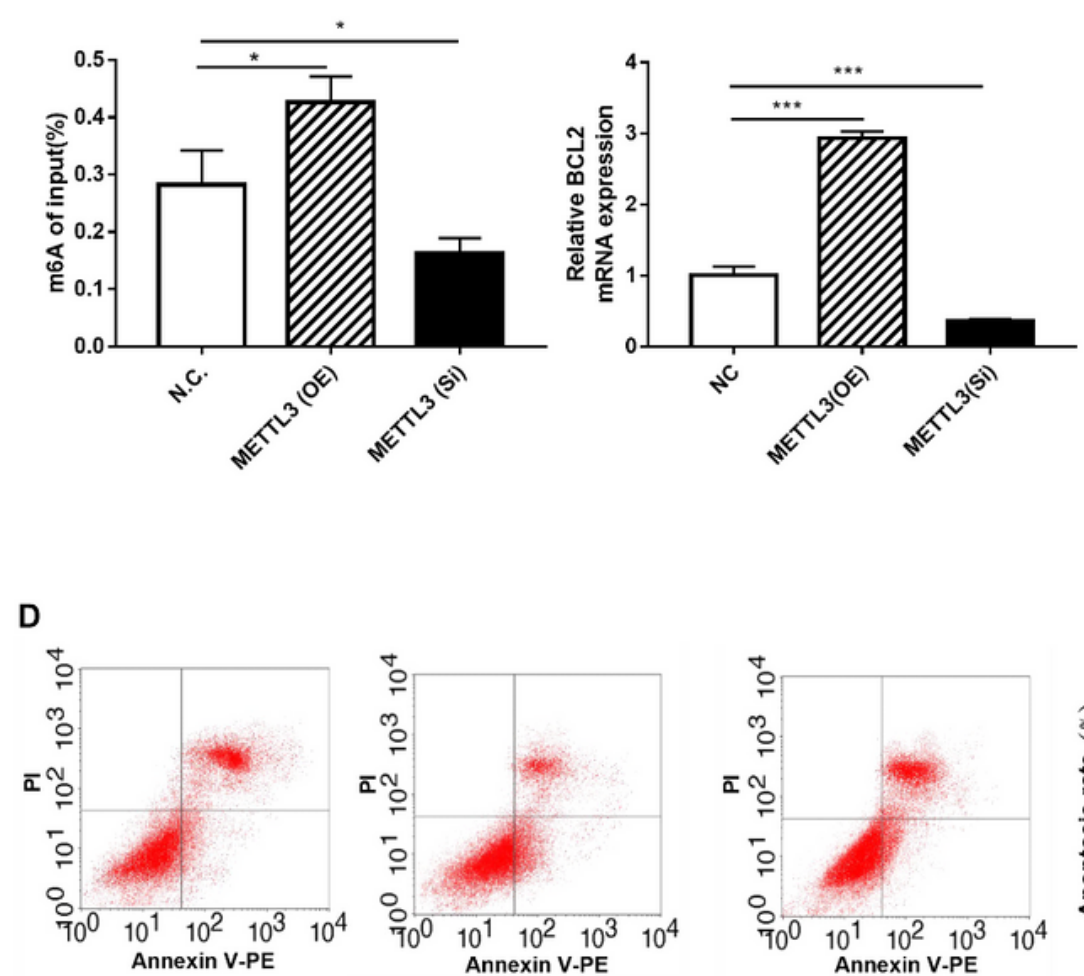

$\mathrm{NC}+\mathrm{CDDP}(\mathrm{IC25})$
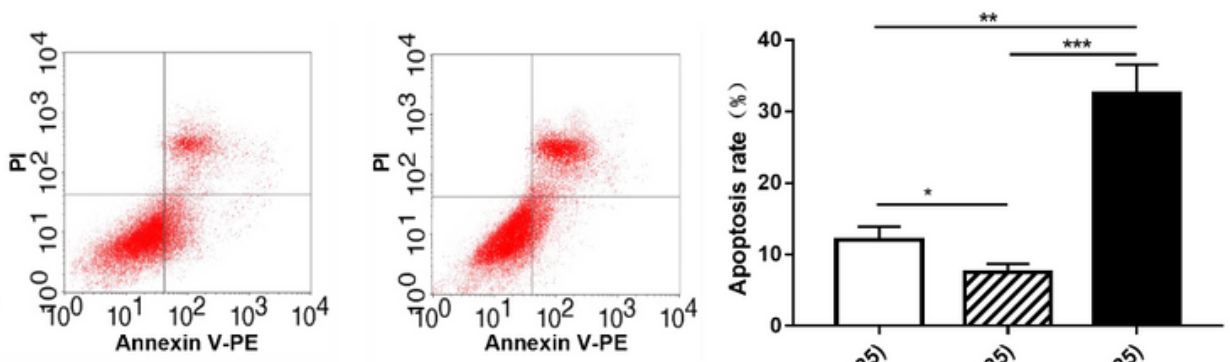

METTL3(OE)+CDDP(IC25) METTL3(OE)+BCL2(Si)+CDDP(IC25)

E
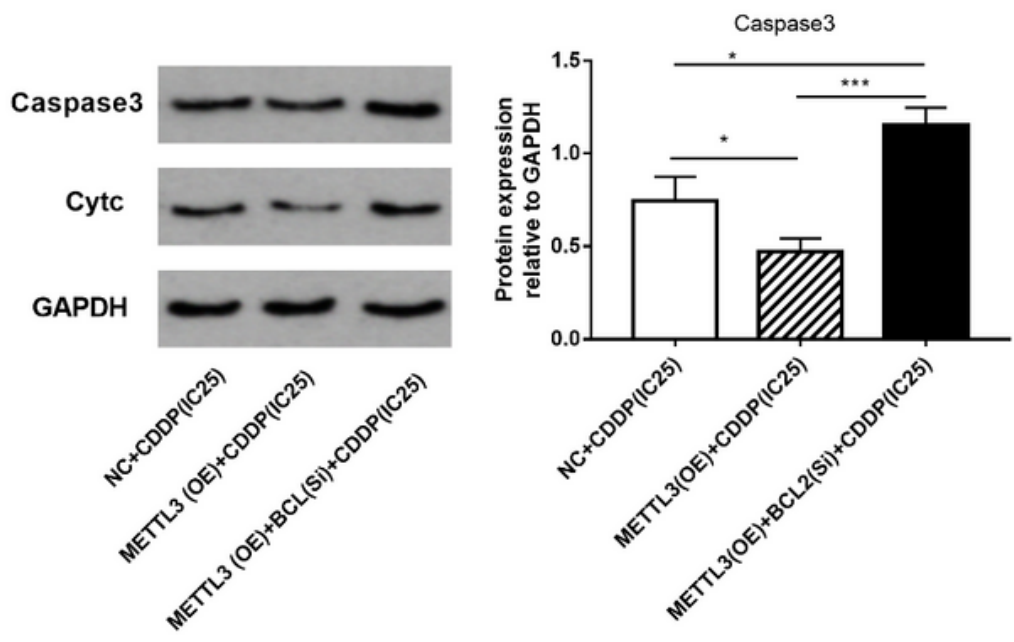

C
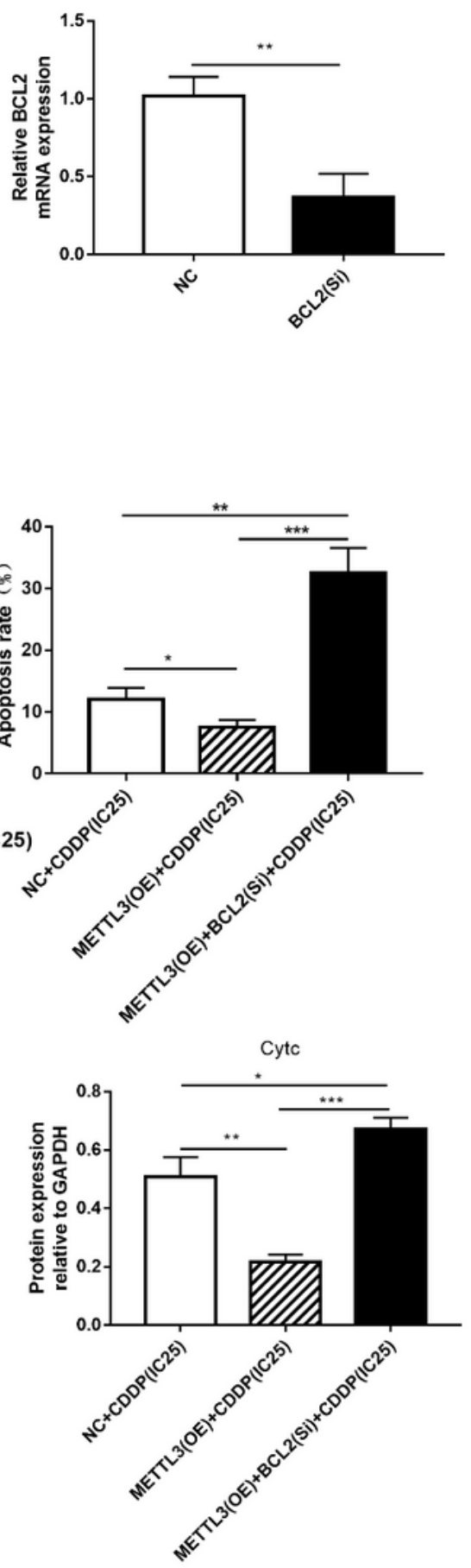

\section{Figure 3}

METTL3 cells enhance the anti-apoptosis ability of TCam-2/CDDP via BCL2. (A, B) m6A modification level of BCL-2 and mRNA expression in total RNA of NC, OE-METTL3 and Si-METTL3 groups. (C) BCL2 mRNA expression in NC and Si- BCL2 groups. (D) Left: Cells in different groups were treated with cisplatin for $72 \mathrm{~h}$, followed by staining with apoptosis analysis kit and then analyzed by flow cytometry. Right: 
Quantitative summary of the apoptosis rate in different groups. (E) Western blot analysis of caspase3 and cytc in different groups. GAPDH served as the internal control.

A

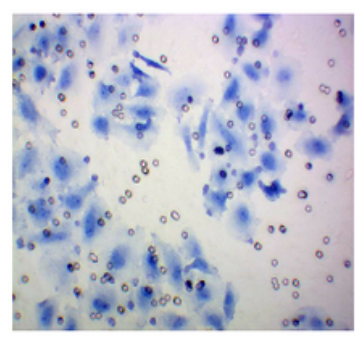

NC

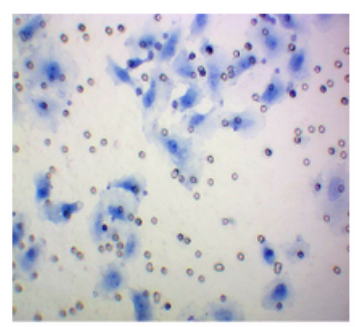

METTL3(Si)

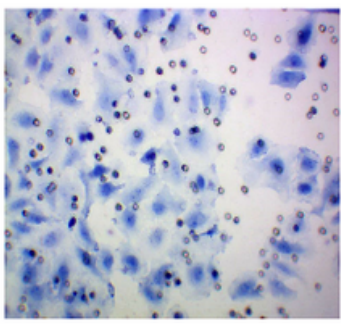

METTL3(OE)

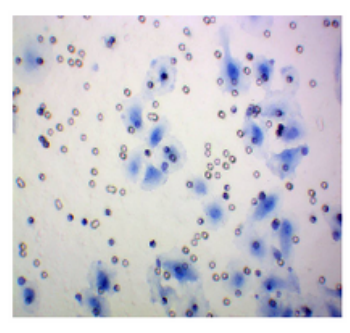

METTL3(OE)+BCL2(Si)
B

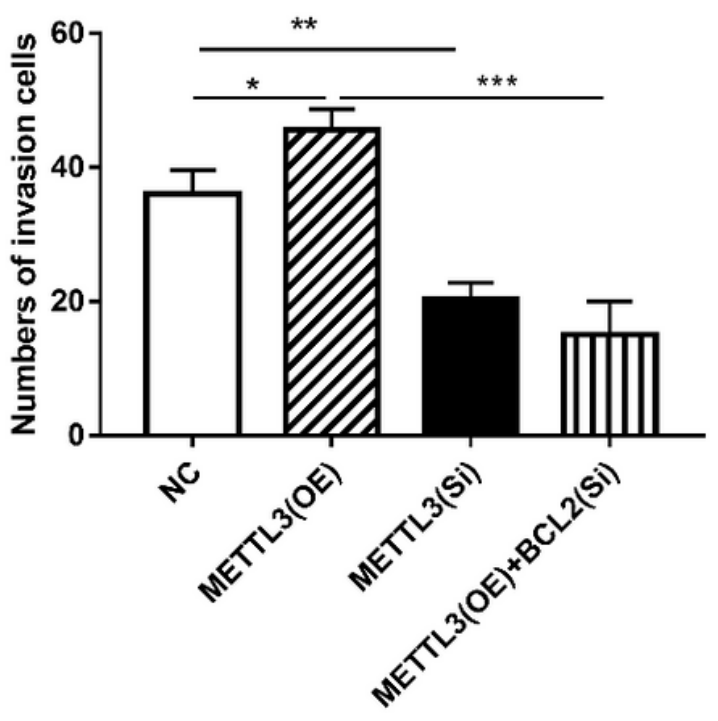

Figure 4

METTL3 enhanced the invasion ability of TCam-2 cells via BCL2. A: Representative images of cells invasion in different groups. B: Quantitative summary of cell numbers in different groups. 


\section{METTL3}

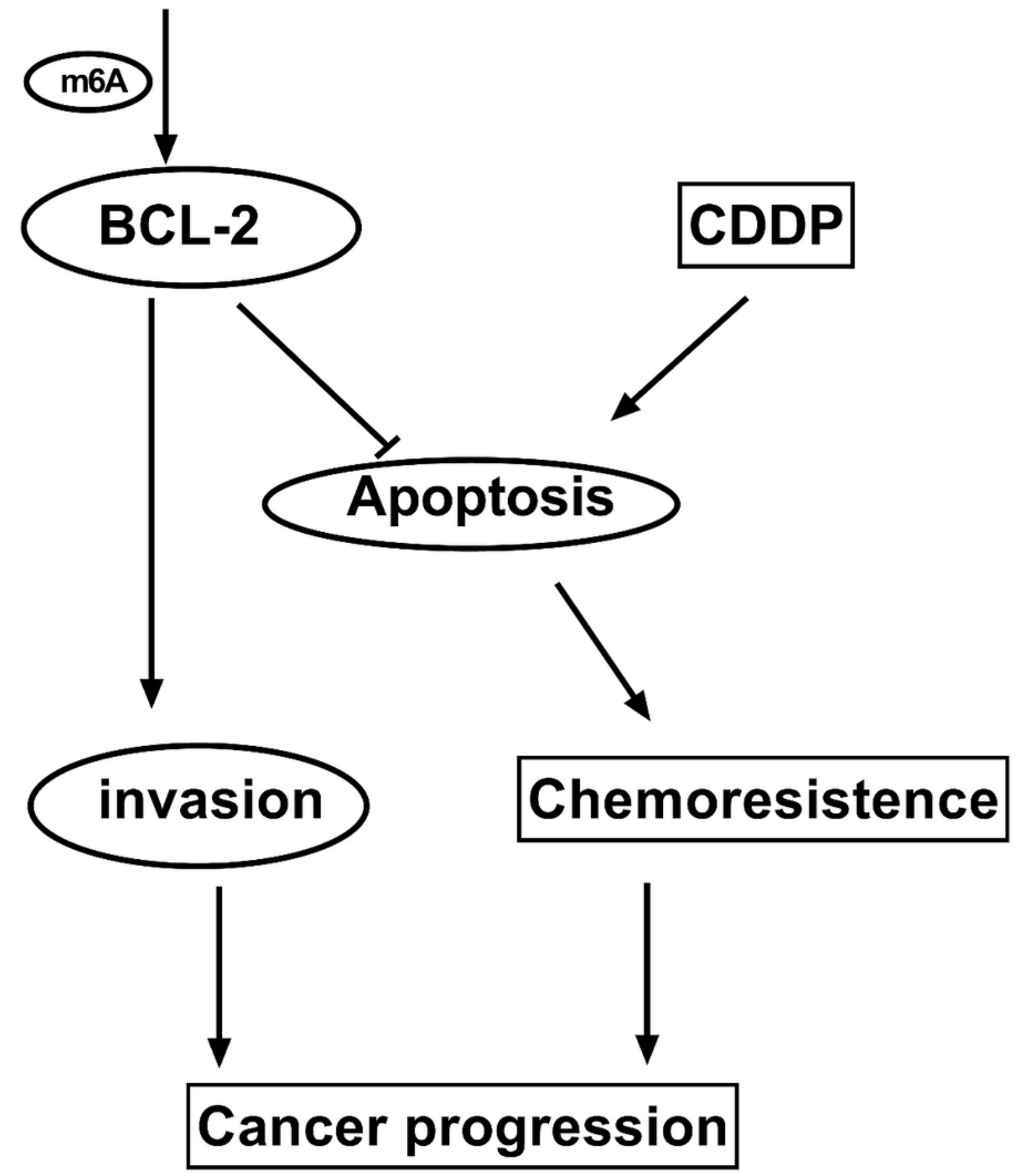

Figure 5

Caption not included with this version. 